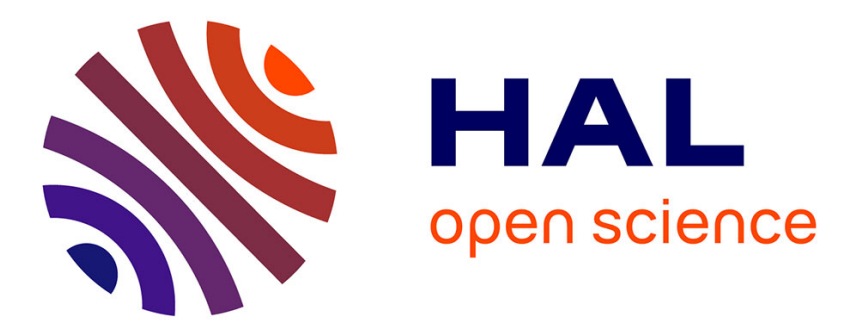

\title{
Du journaliste actionnaire au journaliste salarié. Sociétés de personnels actionnaires et comités d'entreprise dans la procédure de rachat du groupe La Vie-Le Monde \\ Camille Dupuy
}

\section{To cite this version:}

Camille Dupuy. Du journaliste actionnaire au journaliste salarié. Sociétés de personnels actionnaires et comités d'entreprise dans la procédure de rachat du groupe La Vie-Le Monde. L'Homme et la Société, 2013, 189-190 (3), pp.269-304. hal-02279796

HAL Id: hal-02279796

https://hal-normandie-univ.archives-ouvertes.fr/hal-02279796

Submitted on 30 Sep 2019

HAL is a multi-disciplinary open access archive for the deposit and dissemination of scientific research documents, whether they are published or not. The documents may come from teaching and research institutions in France or abroad, or from public or private research centers.
L'archive ouverte pluridisciplinaire HAL, est destinée au dépôt et à la diffusion de documents scientifiques de niveau recherche, publiés ou non, émanant des établissements d'enseignement et de recherche français ou étrangers, des laboratoires publics ou privés. 


\title{
DU JOURNALISTE ACTIONNAIRE AU JOURNALISTE SALARIÉ. SOCIÉTÉS DE PERSONNELS ACTIONNAIRES ET COMITÉS D'ENTREPRISE DANS LA PROCÉDURE DE RACHAT DU GROUPE LA VIE-LE MONDE
}

\author{
Camille Dupuy \\ L'Harmattan | « L'Homme \& la Société »
}

2013/3 n 189-190 | pages 269 à 304

ISSN 0018-4306

Article disponible en ligne à l'adresse :

https://www.cairn.inforevue-l-homme-et-la-societe-2013-3-page-269.htm

Distribution électronique Cairn.info pour L'Harmattan.

(C) L'Harmattan. Tous droits réservés pour tous pays.

La reproduction ou représentation de cet article, notamment par photocopie, n'est autorisée que dans les limites des conditions générales d'utilisation du site ou, le cas échéant, des conditions générales de la licence souscrite par votre établissement. Toute autre reproduction ou représentation, en tout ou partie, sous quelque forme et de quelque manière que ce soit, est interdite sauf accord préalable et écrit de l'éditeur, en dehors des cas prévus par la législation en vigueur en France. Il est précisé que son stockage dans une base de données est également interdit. 


\section{Du journaliste actionnaire au journaliste salarié. Sociétés de personnels actionnaires et comités d'entreprise dans la procédure de rachat du groupe La Vie-Le Monde}

Dans un contexte de crise, où le pouvoir de l'actionnaire est mis en cause pour ses effets destructeurs sur l'emploi et l'investissement, la notion même d'entreprise est interrogée, afin de sortir de la vision d'une société détenue par des actionnaires qui nomment des dirigeants pour aller vers un idéal type de création collective qui implique une participation des salariés aux décisions ${ }^{1}$. Cette vision de l'entreprise repose sur l'idée que celle-ci est avant tout « des gens » (et pas des actionnaires propriétaires) qu'il faut consulter sur les affaires qui les concernent ${ }^{2}$. L'actionnariat salarié apparaît ainsi comme une «alternative à la valeur actionnariale $^{3} »$ dans la recherche de contre-pouvoirs, et est encouragé par la législation, notamment depuis la loi de finances de 2005. Paradoxalement, les salariés du groupe Le Monde ${ }^{4}$ s'engagent dans une voie inverse en 2010, en participant à la vente d'un groupe, initialement dominé par des représentants des salariés, à un trio d'actionnaires externes, devenus

1. Comme le proposent Armand HatchuEl et Blanche SEgRESTin, in Refonder l'entreprise, Paris, Seuil, 2012.

2. Roger BAUdoIn (éd.), L'entreprise : formes de la propriété et responsabilité sociale, Collèges des Bernardins, Éditions Lethilleux, 2012.

3. Antoine REBERIOUX, «Gouvernance d'entreprise et théorie de la firme : quelle alternative à la valeur actionnariale ? », Revue d'économie industrielle, vol. 104, 2003, p. 85110.

4. Le quotidien Le Monde fait partie du groupe Le Monde.

L’homme et la société, $\mathrm{n}^{\circ}$ 189-190, juillet-décembre 2013 
depuis majoritaires dans le capital ${ }^{5}$ (voir repères chronologiques). Comment expliquer l'engagement des salariés dans une transformation apparemment contradictoire avec leur «culte de l'indépendance»?

Historiquement, l'indépendance du Monde ${ }^{6}$, aux yeux de ses journalistes, passe par le contrôle de son capital. Rapidement après sa création en 1944, et dans la mouvance des différents projets qui se succèdent pour doter les entreprises de presse d'un statut qui en ferait des entreprises non nécessairement profitables ${ }^{7}$, les journalistes du Monde cherchent à faire reconnaître une participation des salariés au capital de l'entreprise. Pour « garantir l'indépendance et la continuité du journal », ils demandent dès 1951 à accéder à « une forme juridique » permettant « de voir consacré [leur] droit à la copropriété du journal ${ }^{8}$. À côté des comités d'entreprise, actifs depuis 1945, les journalistes du titre, réunis dans la société des rédacteurs du Monde (SRM), accèdent ainsi, en 1951, à 28,57\% du capital du journal. Les cadres puis les employés rejoignent les journalistes au capital en 1968, sans remettre en cause la position dominante de ces derniers. Entre 1951 et 2010, les salariés du quotidien Le Monde, puis du groupe qui s'est formé autour de lui, demeurent le premier actionnaire de leur entreprise.

Notre hypothèse est que la vente du groupe Le Monde a mis au jour les limites du pouvoir actionnarial (lié aux droits de propriété) aux yeux des salariés eux-mêmes. En effet, ce pouvoir actionnarial a conduit à une focalisation sur le contrôle du capital de l'entreprise, dans une situation de pertes financières chroniques. En imposant aux salariés une vision de l'entreprise à partir d'un diagnostic comptable, il a contribué à occulter les débats sur les contenus mêmes du travail et du produit. Dès lors, l'emploi est apparu comme une variable d'ajustement, en conférant aux comités d'entreprise le soin de mettre en œuvre une politique de réduction d'effectifs, tempérée par des conditions de départ avantageuses. Selon nous, en organisant la mise en vente du journal, les représentants des

\footnotetext{
5. Pour un récit journalistique de cet épisode, cf. Odile BENYAHIA-KOUIDER, Un si petit monde, Paris, Fayard, 2011.

6. Cf. Patrick Eveno, Le Journal Le Monde : une histoire d'indépendance, Paris, Odile Jacob, 2001.

7. Pour plus de détails sur ces projets, cf. Patrick EvENO, L'argent de la presse française des années 1820 à nos jours, Paris, Éditions du CTHS, 2003 ; et Francis SCHWARZ, Les sociétés de rédacteurs en France. Actions et pensées d'un mouvement démocratique pour la presse quotidienne (des origines à nos jours), Thèse de doctorat en sciences de l'information et de la communication, soutenue le 16 avril 1991 à l'Université Michel Montaigne - Bordeaux III.

8. Extrait d'une motion adressée aux actionnaires en 1951, reproduite in Jean SCHWEBEL, La presse, le pouvoir et l'argent, Paris, Éditions du Seuil, 1968, p. 100.
} 
salariés au capital de l'entreprise ont engagé un processus de redécouverte du pouvoir salarial porté par les comités d'entreprise (lié au droit du travail). Cela implique pour les représentants des salariés une évolution dans leurs registres d'action juridique, en passant d'un jeu sur le droit des sociétés à un usage plus intense du droit du travail.

Cette transition d'un pouvoir actionnarial à un pouvoir salarial fait apparaître une « capacité politique ${ }^{9}$ » nouvelle des salariés, entendue comme le pouvoir de peser sur les choix engageant l'avenir de l'entreprise et de ses membres. Cela conduit à ne pas limiter la participation des salariés ${ }^{10}$ à leur entrée dans le capital de l'entreprise, en prenant également en compte les garanties qu'apporte le droit du travail à une expression collective des salariés. Dans la continuité des travaux de Max Weber sur la presse $^{11}$, nous proposons de regarder le journaliste en tant que salarié dans une entreprise capitaliste. Le pouvoir salarial repose sur les apprentissages qui s'opèrent à partir d'une mobilisation d'un droit qui, sans initiative des acteurs, reste limité à la gestion des «conséquences sociales » des choix actionnariaux. Un salarié dispose d'un pouvoir lorsqu'il a la $c a$ pacité de mobiliser et d'utiliser son droit de manière libre (positive).

Nous étudions le moment où le cadre institutionnel de l'entreprise change, avec le passage d'un actionnariat salarié à un actionnariat et un salariat classiques. Dans une perspective institutionnaliste, nous considérons le cadre institutionnel comme un outil d'analyse de la relation salariale $^{12}$. Nous envisagerons l'activité des deux instances représentatives du personnel : les sociétés de personnels actionnaires d'une part, et les comités d'entreprise d'autre part. Nous porterons une attention particulière à la manière dont les représentants des salariés dans ces deux types d'instance

9. Définie par Claude DIDRY, in «L'approche par les capacités comme registre des restructurations : un nouveau regard sur l'entreprise et le contrat de travail ? », Revue française de sociologie, 2013, 54-2, p. 335-367.

10. Cf. le rapport de Salima BenHAMOU, « Améliorer la gouvernance d'entreprise et la participation des salariés », Rapports et documents du Centre d'analyse stratégique, Paris, La Documentation française, 2010.

11. Max WEBER, Rapport préliminaire pour une proposition d'enquête sur la sociologie de la presse, 1910, traduit en annexe de Gilles BASTIN, « La presse au miroir du capitalisme moderne. Un projet d'enquête de Max Weber sur les journaux et le journalisme », Réseaux, $\mathrm{n}^{\circ} 2001 / 5$, p. 172-208.

12. On utilise la notion de « relation salariale » qui est plus large que celle de « rapport salarial » qui se centre sur les questions de pouvoir et de domination d'un groupe sur l'autre, et qui est plus précise que «relation d'emploi » en se centrant sur les caractéristiques de l'acteur en tant qu'il est salarié (et pas seulement en emploi). Cf. les définitions de Jean-Louis RENOUX, Les régulations de la relation d'emploi dans les médias français, thèse de doctorat en sociologie, Université Paris Dauphine, Paris, 2011. 
mobilisent leurs droits comme des références ou des points d'appui, pour peser sur le processus de recapitalisation en sortant de la question du contrôle du capital pour aller vers une interrogation sur le devenir des entreprises du groupe et de leurs salariés. In fine, ce cas interroge les capacités politiques des salariés dans un contexte de corporate governance ${ }^{13}$.

Notre étude s'appuie sur trois types de matériaux. Tout d'abord, une revue des articles de presse parus sur cette question dans Le Monde et Les Échos entre mars et novembre 2010 nous a permis de constituer une chronologie segmentée en quatre phases ${ }^{14}:$ l'avant recapitalisation ; les candidatures à la reprise ; la négociation exclusive avec l'offre retenue ; le rachat et ses conséquences. Ensuite, nous avons mené treize entretiens semidirectifs avec les acteurs engagés dans ce rachat. Tout d'abord, nous avons rencontré les représentants des salariés du quotidien (huit) et du groupe (quatre à Courrier International et au Monde Interactif) qui ont des mandats dans une société de personnels ou un syndicat de chaque catégorie (journalistes, employés et cadres). Nous avons complété en rencontrant un représentant de la direction et des actionnaires. Les enquêtés sont des hommes (pour douze des treize entretiens), avec un âge moyen de 50 ans au quotidien, 30 ans pour les autres titres du groupe ${ }^{15}$. Ces entretiens ont été conduits entre juin 2010 et avril 2011, à des moments différents du processus ${ }^{16}$, ce qui peut poser des problèmes méthodologiques quant à la déformation de la parole des enquêtés. Pour résoudre ce problème, nous avons enfin eu accès à une importante documentation écrite qui retrace les étapes des négociations autour de la recapitalisation (procès-verbaux des réunions du comité d'entreprise, rapports des experts du comité d'entreprise, communiqués des sociétés de personnels ou de l'intersyndicale, propositions des candidats, protocoles d'accords, etc.).

Pour comprendre comment les salariés ont accepté cette transition vers un nouveau mode de gouvernance, le rôle qu'ils y ont joué et les conséquences sur la relation salariale, on étudiera le déroulement du processus dans l'ordre chronologique des événements : la mise en vente du groupe Le Monde, puis la négociation de nouveaux droits pour les salariés et enfin l'arrivée de nouveaux «patrons».

\footnotetext{
13. Bernard BAUDRY, Économie de la firme, Paris, La Découverte, coll. « Repères », 2003.

14. Cf. Repères chronologiques p. 303.

15. Cet écart s'explique facilement par l'écart de l'âge moyen dans les rédactions.

16. Nous spécifions la date de l'entretien lorsque cela a du sens dans la démonstration, sinon, nous essayons d'anonymiser au maximum l'identité des enquêtés.
} 


\section{I - Le Monde à vendre? Quand les salariés s'accrochent à leur groupe}

Les importantes difficultés financières du groupe ont conduit à l'éviction de son dirigeant historique, Jean-Marie Colombani, en 2008. Mais deux ans plus tard, en l'absence de redressement et sous le poids des dettes accumulées, c'est la pérennité même du groupe qui est mise en question. La voie initiale de la recapitalisation est rapidement écartée, tout comme celle du redressement judiciaire et du démantèlement du groupe par la vente des titres qui le constituent. La voie privilégiée est celle d'une mise en vente du groupe, dans sa totalité, à d'éventuels repreneurs. Cette démarche, engagée par les différents canaux de représentation des salariés (sociétés de personnels actionnaires, comités d'entreprise), traduit un attachement des salariés à leur groupe, ainsi que le besoin de renouveler les rapports entre salariés, dirigeants et actionnaires.

\section{Un groupe à recapitaliser?}

Le groupe Le Monde doit son existence au chantier qu'entreprend J.M. Colombani dès la fin des années 1990. Comme il l'explique, suite à la crise de 1994, « la construction du groupe n'avait qu'un seul but : inscrire Le Monde dans un périmètre économique suffisamment large pour que son indépendance soit préservée ${ }^{17}{ }$.

La constitution juridique du groupe est confiée à Alain Minc, mais peu d'efforts sont menés pour créer une cohérence interne au groupe, dont l'unique objet est de gérer les dettes du quotidien. Les titres rachetés, comme dans le cas du Midi Libre et des Cahiers du Cinéma (revendus depuis) ou les titres des Publications Vie Catholique ( $L a$ Vie, Télérama, etc.) sont conçus comme des «vaches à lait» (entretien). Les salariés de Télérama ont particulièrement pâti de leur intégration dans le groupe, puisque suite à la vente de leur immeuble et l'absorption de la trésorerie du titre, celui-ci est mis en gage contre un prêt bancaire de 25 millions d'euros, en mars 2009. Cette situation explique l'«état de défiance ahurissant » (entretien) des salariés du groupe envers les journalistes du quotidien.

Après les achats et les cessions des années 2000, au rythme des besoins de la société éditrice du Monde, le groupe Le Monde emploie avant la recapitalisation 1254 salariés en contrat à durée indéterminée (566 journalistes, 331 cadres, 345 ouvriers et employés) ${ }^{18}$, et se divise en trois pôles : un pôle presse quotidienne nationale (Le Monde, l'imprimerie, Le

17. Jean-Marie Colombani, Le Monde du 9 novembre 2010.

18. Ces chiffres correspondent à la situation en octobre 2010. 
Monde publicité), un pôle magazines et livres (Télérama, Courrier International, Le Monde diplomatique, Malesherbes publications, Publicat...) et un pôle interactif (Le Monde Interactif, I-Régie, le Post). Les résultats du groupe sont en constante dégradation depuis 2001. À la veille de la recapitalisation, le résultat opérationnel du groupe est déficitaire, de plus de dix millions d'euros. En cause, les mauvais résultats du pôle presse quotidienne nationale et du pôle magazines et livres. Le pôle Interactif est le seul dont le résultat opérationnel soit bénéficiaire en 2009 et 2010. Le groupe est très endetté, puisqu'au prêt de 25 millions gagé sur Télérama, s'ajoute notamment le remboursement d'obligations remboursables en actions (ORA) d'un montant de 35 millions, et la nécessité de moderniser l'imprimerie $^{19}$. Le 28 mars 2010, les membres du directoire du groupe, Éric Fottorino et David Guiraud, annoncent aux représentants des salariés, réunis au sein du comité de groupe, la nécessité d'une recapitalisation à hauteur de 50 à 60 millions d'euros, besoins réévalués à 100 millions d'euros au cours du printemps 2010.

Alors que les salariés constituent le premier actionnaire du groupe (notamment par l'intermédiaire de la SRM), cette nouvelle recapitalisation leur ferait perdre cette place en diluant leurs droits de propriété. Comment expliquer, malgré cette remise en cause de leur pouvoir actionnarial, l'accord des salariés avec cette nécessité à recapitaliser ? Pour expliquer la situation présente, Éric Fottorino (le directeur au moment de la recapitalisation) insiste sur « les carences de la gestion passée » (de Jean-Marie Colombani) qui a laissé l'entreprise dans une situation économique et financière désastreuse : "la vérité des chiffres est apparue dans sa froideur ». La direction actuelle aurait « assaini sa gestion sans renoncer à ses ambitions éditoriales » mais ne pouvait revenir d'une pareille situation ${ }^{20}$. En réponse à l'article d'Éric Fottorino, Jean-Marie Colombani tente au contraire d' «endogénéiser » les raisons qui ont mené à cette situation, en mettant en avant «trois années de déconstruction [du groupe] » par les dirigeants et en faisant porter la responsabilité à la direction d'Éric Fottorino $^{21}$. Cette stratégie échoue et, prenant les chiffres à témoin, les directeurs et les salariés partagent l'idée que la recapitalisation est inévitable. Grâce à ces rhétoriques «impersonnalisées et exogénéisantes », l'argu-

19. Les différents rapports d'expertise précédemment cités font mention de ces chiffres.

20. Éric FotTorino, « Ecrire une nouvelle page », Le Monde, 3 novembre 2010.

21. Dans Le Monde du 9 novembre 2010, en réponse à l'article d'Éric Fottorino (Le Monde, 3 novembre 2010). 
mentaire n'est pas déconstruit ${ }^{22}$. Tous sont pris dans l'urgence puisque le groupe serait en cessation de paiement le 10 juillet sans l'arrivée d'argent frais. La « réunion zéro » du comité d'entreprise, le 18 juin 2010, s'ouvre ainsi sur le calendrier, et les contraintes de temps sont rappelées tout du long : « calendrier contraint », « calendrier très serré », « gagner du temps », « tenir cette échéance ». Dans tous les entretiens menés avec des salariés de la société éditrice du Monde, les enquêtés mettent en avant cette obligation à recapitaliser.

«Le groupe était endetté, surendetté, on ne pouvait pas s'en sortir seul.» (Entretien avec un journaliste, mars 2011)

«On était tous d'accord pour qu'elle [la recapitalisation] ait lieu, parce que depuis 2002 on perd de l'argent. [...] Donc on est arrivé à un moment de nonretour. » (Entretien avec un cadre, mars 2011)

«Il faut se rappeler l'histoire, avant juin, Le Monde était en situation de dépôt de bilan. On n'avait pas un choix fou. » (Entretien avec un employé, mars 2011)

Ce discours n'est pas seulement de l'ordre de la justification postérieure au rachat (pourquoi on a vendu nos parts ?) puisqu'il se retrouve dès le début des négociations, comme le montre cet extrait d'entretien réalisé au printemps 2010 :

«Moi je pense que la recapitalisation est inéluctable car ça voudrait dire refuser de traiter les problèmes qui sont les nôtres, qu'on refuse de traiter depuis des années. » (Entretien avec un journaliste)

Pourtant, plusieurs réserves sont émises à propos du processus de recapitalisation. Tout d'abord, si l'expert du comité d'entreprise confirme l'impasse financière (cessation de paiement le 10 juillet), il établit cependant que la situation n'est pas «si catastrophique » et qu'il existe toujours des «marges de manœuvre». «Quelle est l'alternative?» demande le président de la société des rédacteurs du Monde (SRM), premier actionnaire du groupe. "C'est le dépôt de bilan ou la vente d'actifs » ${ }^{23}$. C'est justement l'alternative proposée par Jean-Michel Dumay (ancien président de la SRM) : déposer le bilan pour épurer les dettes puis négocier avec un repreneur. Cette solution a rapidement été écartée :

« Ça réglait notre problème financier mais ça voulait dire qu'on livrait nos destins aux mains du tribunal de commerce... » (Entretien avec un journaliste)

22. Comme dans les argumentaires justifiant les plans sociaux, exposés par Tristan BOYER, «Déconstruction du projet de licenciement », Revue de l'IRES, n 47, 2005/1, p. $175-192$.

23. Entretien publié sur le site Mediapart le 17 avril 2010. 
Même si l'alternative du redressement judiciaire et celle du démantèlement du groupe sont évoquées, le diagnostic partagé d'un besoin de financement conduit les représentants des salariés à accepter le principe d'une recapitalisation du groupe dans son ensemble. Ainsi, en dépit du caractère inéluctable que constitue le besoin de financement pour échapper à une cessation de paiement attendue pour juillet 2010, le refus du redressement judiciaire traduit un choix de l'ensemble des acteurs et, parmi eux, de l'ensemble des salariés, en faveur d'un maintien du groupe dans sa configuration actuelle.

\section{Recapitaliser ou vendre?}

Étudier la genèse de l'actionnariat du groupe est un préalable nécessaire pour comprendre la dynamique de l'entreprise Le Monde ${ }^{24}$. Le Monde est créé en décembre 1944 et placé sous la direction d'Hubert Beuve-Méry. Jusqu'en 1951, le capital est réparti entre neuf porteurs de parts indépendants (dont le directeur). Le directeur du journal est très attaché à l'indépendance des journalistes: "méfiez-vous de l'argent restera son cri de guerre jusqu'à la fin de sa vie ${ }^{25} »$. Début 1951, un désaccord éclate entre Hubert Beuve-Méry et deux autres membres du comité de rédaction, qui provoque sa démission. La rédaction se soulève. Les négociations aboutissent rapidement à la création de la société des rédacteurs du Monde (SRM) le 12 décembre 1951. Elle représente la communauté des rédacteurs, qui se constitue en société anonyme et reçoit $28,57 \%$ des parts du journal (acquisition collective des rédacteurs). Ce pourcentage leur permet de disposer d'une minorité de blocage sur les décisions extraordinaires (prises aux trois quarts des voix) qui sont «les plus importantes et les plus susceptibles d'affecter le destin de l'entreprise comme le leur propre ${ }^{26} \gg$. Cela s'inscrit dans un moment de réflexion, lancé par le Conseil national de la Résistance, qui, dans Le Cahier bleu, propose un statut de l'entreprise de presse différent du statut des entreprises commerciales, afin que la presse ne retombe pas sous la domination de l'argent. La SRM va être un modèle pour les journalistes des autres entreprises de presse, qui vont à leur tour constituer des sociétés de journalistes à partir

24. Pour une histoire complète, cf. Patrick Eveno, Histoire du journal Le Monde (1944-2004), Paris, Albin Michel, 2004.

25. François Simon, Le journaliste. Dans les pas d'Hubert Beuve-Méry, Paris, Arléa, 2005 , p. 75 .

26. Jean SCHWeBel, La presse, le pouvoir et l'argent, Seuil, 1968, p. 103. 
de $1965^{27}$. En 1968, les parts de la SRM seront portées à $40 \%$ du capital tandis que $10 \%$ des parts sont accordées à deux nouvelles sociétés de personnels, celle des employés et celle des cadres. Ainsi, « le véritable propriétaire du journal, c'est la rédaction ${ }^{28} »$.

Le Monde ouvre son capital pour la première fois en 1985 à des dons privés. Un appel public à l'épargne est mené par la Société des lecteurs, nouvellement créée sous la direction d'Alain Minc, et réunit près de 15 millions de francs. C'est ensuite au tour d'entreprises privées d'entrer dans le capital en 1986, avec 21 souscripteurs (Saint Gobain, BNP, L'Oréal...) réunis dans Le Monde Entreprises (11 millions de francs). Jean-Marie Colombani accède à la direction du Monde en 1994. La SARL Le Monde est transformée en une Société anonyme avec un directoire et un conseil de surveillance présidé par Alain Minc. Le groupe La Vie-Le Monde voit le jour en 2001 et s'organise en une structure tripartite imaginée par Alain Minc (figure 1): Le Monde Partenaires Associés (LMPA), société mère du groupe détient $60,40 \%$ du capital du Monde SA (LMSA), et se partage entre les actionnaires internes et les actionnaires externes ou partenaires ; LMSA est la structure qui accueille les nouveaux actionnaires lors des recapitalisations et détient $94,70 \%$ de la Société éditrice du Monde (SEM) ; la SEM est la société éditrice du quotidien Le Monde et la structure porteuse de l'ensemble des filiales du groupe.

Cette structure permet, selon les acteurs, de soumettre le pouvoir économique au pouvoir politique, puisqu'elle rend possible des recapitalisations sans modifier la répartition des droits de vote au sein de LMPA qui contrôle LMSA. Ainsi, lors de l'augmentation de capital qui voit l'arrivée des groupes Lagardère et Prisa en 2004, les prises de part se font au niveau de LMSA et non de LMPA, laissant la majorité aux « actionnaires internes » c'est-à-dire aux salariés réunis dans des sociétés de personnels (rédacteurs du Monde, cadres du Monde, employés du Monde, personnels des Publications Vie Catholique). La nouvelle recapitalisation envisagée en 2010 est d'une autre nature. Contrairement à la précédente, même si elle est prévue au niveau de LMSA et non de LMPA, elle ferait perdre aux actionnaires internes le contrôle du groupe, en diluant la part de LMPA dans LMSA (figure 2). Plus qu'une recapitalisation, c'est d'une

27. Pour une histoire des SDJ, cf. Camille DuPuY, «Les sociétés de journalistes d'hier et d'aujourd'hui : l'héritage de Jean Schwœbel et de la société des rédacteurs du Monde », in Patrick BeZIERs, Patrick Eveno et Pascale Goetschel (dir.), Rassembler, organiser, protéger. La protection sociale des métiers du spectacle et des médias, Paris, Éditions Jacob-Duvernet, 2012.

28. Propos prêtés à Hubert Beuve-Méry, in François SimON, Le journaliste. Dans les pas d'Hubert Beuve-Méry, op. cit., p. 131. 
vente dont il s'agit désormais, accompagnée d'une perte du pouvoir actionnarial pour les salariés via les sociétés de personnels actionnaires. Ainsi, comme l'explique ce salarié :

«Puisqu'on est au chapitre sémantique, vous voyez que partout on vous parle de nouveaux actionnaires, mais ce ne sont pas des nouveaux actionnaires, ce sont des nouveaux propriétaires... » (Entretien avec un délégué syndical du Monde.)

Figure 1 : Structure de l'actionnariat du groupe Le Monde avant 2010

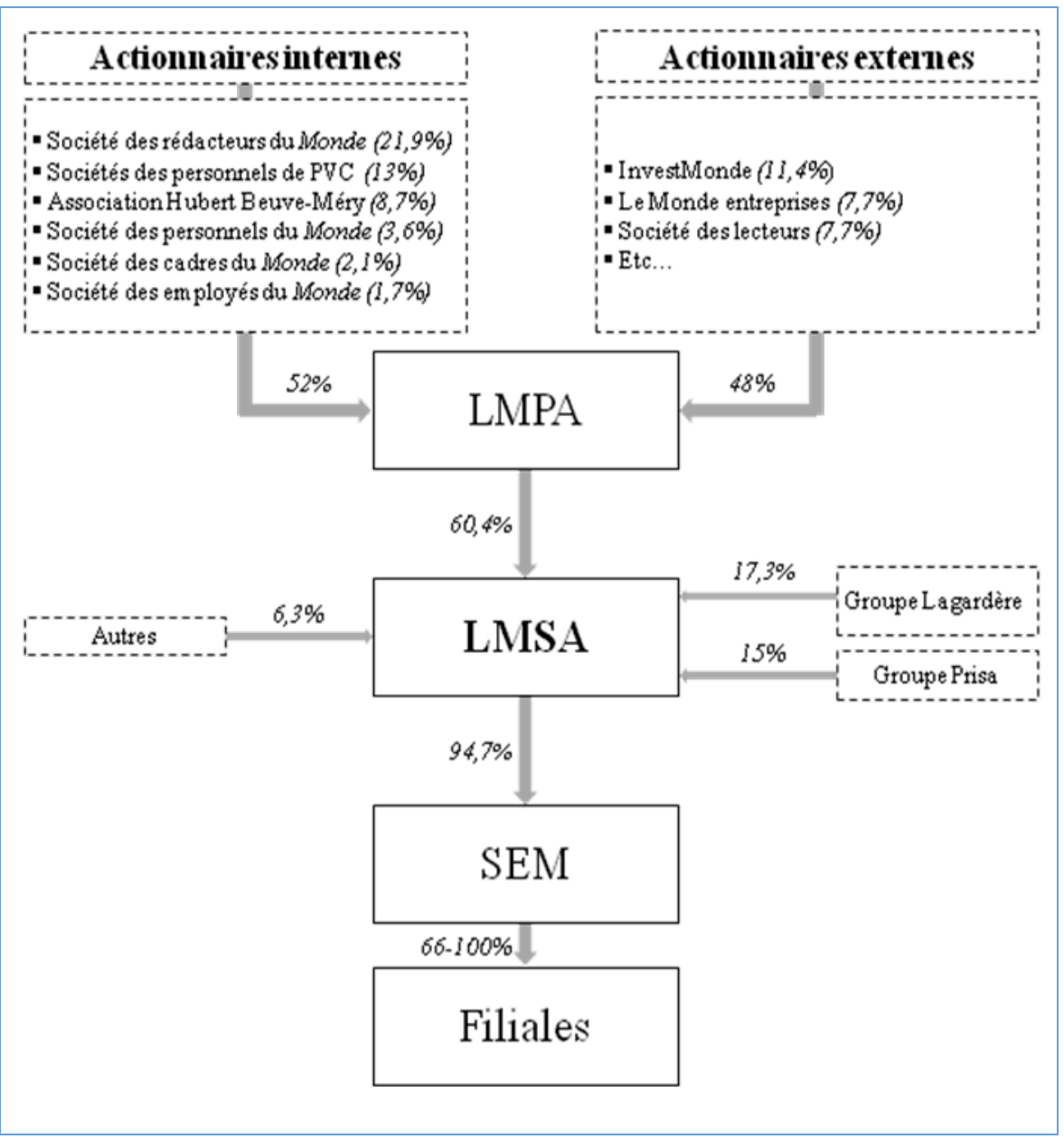


Figure 2 : Avant et après recapitalisation

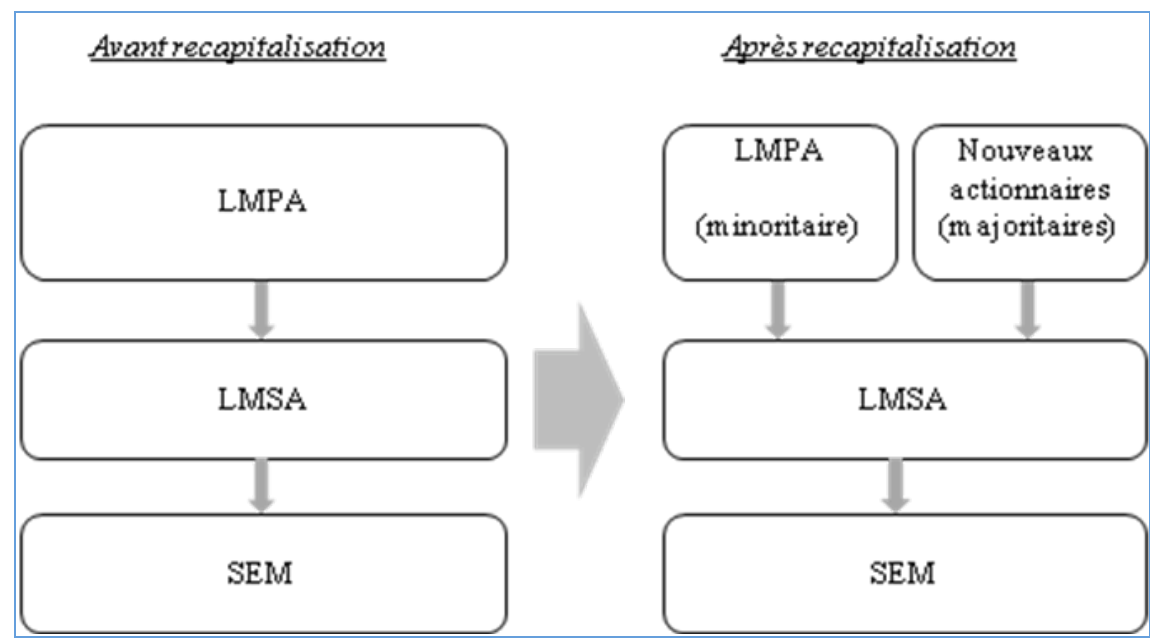

Comment expliquer que les salariés actionnaires acceptent de céder leurs droits de propriété ? Ils renoncent à ce modèle de gouvernance d'entreprise dans lequel les salariés sont les actionnaires. Selon eux, ce système de cogestion est «à bout de souffle » et doit être renouvelé. Un délégué syndical décrit « un système de cogestion où tout le monde était un peu empêtré » (entretien). En effet, l'existence d'intérêts contradictoires entre les différentes postures (salariés actionnaires et salariés en entreprise) pose problème. Un ancien président de la SRM (1977-1981) explique :

«On peut imaginer le problème qui se présente lorsqu'en tant que président de la société des rédacteurs, je vais essayer d'appuyer une décision de la gérance et que, par derrière, le syndicat auquel j'appartiens - j'ai payé une cotisation me dit cela ne va pas aller, nous n'allons pas l'accepter et à l'occasion nous allons provoquer une grève.» (Entretien)

Cette confusion pousse certains syndicalistes à ne pas participer aux sociétés de personnels ${ }^{29}$. La mise en vente du groupe correspond donc à la faillite de ce système d'actionnariat-salariat dominé par les journalistes du Monde, à l'œuvre entre 1951 et 2010.

29. Cette position est notamment celle des salariés de l'imprimerie, qui malgré le poids qu'ils représentent dans le groupe (233 salariés soit presque $20 \%$ des effectifs) n'ont jamais souhaité se constituer en société de personnels actionnaires. 


\section{Le rachat du Monde}

Le rachat du groupe Le Monde se fait avec l'assentiment de la direction et des salariés. Quelle place tient chacun des acteurs dans ce processus ? La direction tente de mettre la main sur la discussion en rencontrant les candidats à la reprise sans en informer les salariés. Un communiqué de l'intersyndicale, le 25 mai 2010, dénonce ainsi « la plus totale opacité » de la procédure. Le directoire cède la place de négociateur aux salariés au mois de juin, prenant conscience que le directoire fait « des recommandations indicatives » mais, « au final, c'est aux personnels de cette maison qu'il revien[t] de se prononcer sur le nom et le projet du futur investisseur $\gg^{30}$, puisqu'ils disposent de 8 sièges sur 20 au conseil de surveillance du groupe (tableau 1).

Tableau 1 : Répartition des sièges au conseil de surveillance de LMPA

\begin{tabular}{|c|c|c|c|c|c|}
\hline $\begin{array}{l}\text { Actionnaires } \\
\text { externes }\end{array}$ & 10 & & & & \\
\hline \multirow{6}{*}{$\begin{array}{l}\text { Actionnaires } \\
\text { internes }\end{array}$} & \multirow{6}{*}{10} & \multirow{4}{*}{$\begin{array}{l}\text { Société des personnels du } \\
\text { Monde }\end{array}$} & \multirow{4}{*}{5} & Journalistes & 2 \\
\hline & & & & Employés & 1 \\
\hline & & & & Cadres & 1 \\
\hline & & & & Personnels & 1 \\
\hline & & $\begin{array}{c}\text { Société des personnels des } \\
\text { autres titres }\end{array}$ & 3 & & \\
\hline & & $\begin{array}{c}\text { Association } \\
\text { Hubert Beuve-Méry }{ }^{31}\end{array}$ & 2 & & \\
\hline
\end{tabular}

La SRM se positionne comme l'interlocuteur privilégié, en agitant la menace «de faire capoter la recapitalisation ${ }^{32}$ » par son «droit de veto sur les opérations de recapitalisation ${ }^{33}$ » au conseil de surveillance du groupe. On voit ici comment les règles sont utilisées de manière stratégique par les acteurs dans l'espoir d'influer sur le cours de l'action. Le président de la SRM nous avouera, à l'issue du processus de recapitalisation, avoir joué sur cette ambigüité :

30. Éric FotTORINO, « À nos lecteurs », éditorial du Monde du 4 juin 2010.

31. Elle réunit les actionnaires historiques.

32. Gilles Van Kote, président de la SRM dans Les Échos du 12 avril 2010.

33. Gilles Van Kote, président de la SRM dans Mediapart du 17 avril 2010. 
«La SRM avait-elle un droit de veto sur la recapitalisation au sein du conseil de surveillance de LMPA?

«Ça a été une confusion qu'on a entretenue nous-mêmes car ça nous arrangeait. Dans les statuts de LMPA, il est dit qu'il y a un droit de veto de la SRM en cas de cession des parts mais là il n'y a pas de cession de parts, c'est une augmentation de capital. » (Entretien avec le président de la SRM)

Cependant, la SRM ne bénéficie pas d'assez de pouvoir pour disposer à elle seule d'un crédit suffisant dans les négociations.

«Il fallait accepter que ceux qui étaient sur une position dominante, descendent de cette position et acceptent de mettre en commun, de mutualiser avec d'autres. » (Entretien avec le président de la SRM)

Ainsi, à l'initiative de la SRM et d'après l'idée de la société des lecteurs, l'ensemble des sociétés de personnels du groupe ${ }^{34}$ s'agrègent dans un «pôle d'indépendance », dans une démarche « fédératrice ».

« Le pôle d'indépendance a émergé en quelques mois. On a commencé à évoquer ce nom au mois d'avril. [...] La démarche qui était la nôtre était de fédérer nos forces. » (Entretien avec le président de la SRM)

Le président de la SRM n'est pas le seul à s'investir dans les discussions. Les autres membres du pôle d'indépendance négocient également avec les candidats. Par exemple, le président de la société des journalistes de Courrier International raconte :

«Moi ce que j'ai fait, en travaillant avec les sociétés de personnels de Télérama et des PVC [Publications Vie Catholique], on a préparé tout ce qui était prise de contact avec les candidats à la reprise. On a fait par exemple un déjeuner au Nouvel Obs avec Perdriel et Oliviennes. On est aussi allé voir Bergé et Pigasse. » (Entretien)

On voit se constituer ici un collectif salarial large, dans lequel, à côté de la SRM, les représentants des autres titres tentent de faire entendre leur voix.

Ce sont donc les instances de représentation des salariés dans le capital (par l'intermédiaire du pôle) qui prennent en main la vente du groupe. Les sociétés de personnels suscitent des candidatures et négocient avec les candidats des garanties d'indépendance.

« [On a discuté] directement avec les sociétés de personnels, en tant qu'actionnaires. Les dirigeants n'étaient pas actionnaires. » (Entretien avec un représentant des actionnaires)

Les sociétés de personnels cherchent à négocier la conservation de certains droits, attachés au pouvoir actionnarial, malgré la dilution des droits

34. Cf. tableau 1. 
de propriété, qui seraient réalloués collectivement au pôle. Par exemple, avant la recapitalisation, la SRM dispose d'un droit de veto sur le président du directoire du groupe, et sur certaines opérations (cessions de parts par exemple), droits qui sont amenés à disparaître avec la vente du groupe. Les salariés entendent garder un certain nombre de ces prérogatives et réclament «de nouveaux garde-fous » collectifs. Ils demandent à participer à la définition du nouveau modèle de gouvernance pour maintenir des garanties d'indépendance et conforter leur position dans le groupe.

Plusieurs projets sont débattus entre avril et juin 2010. Le 21 juin 2010, date limite de dépôt des offres, deux trios d'investisseurs se portent candidats au rachat du Monde. Le premier trio, «POP », est composé de Claude Perdriel (Le Nouvel Observateur), du groupe Prisa (groupe de presse espagnol actionnaire minoritaire du groupe) et d'Orange (opérateur de télécommunication). Le second projet, «BNP», est porté par Pierre Bergé, Mathieu Pigasse (Les Inrockuptibles) et Xavier Niel (Free).

Dans cette première phase, les sociétés de personnels restent focalisées sur la question du contrôle capitalistique et managérial du groupe, sans envisager les dimensions autour de l'emploi et du produit. Les Comités d'entreprise sont absents de ces discussions qui n'engagent que les salariés actionnaires.

\section{II - Du pouvoir actionnarial au pouvoir salarial : l'entrée en scène des comités d'entreprise}

Dans cette seconde phase, les salariés du groupe sont invités à entrer en négociation avec des repreneurs pour conclure un accord sur la vente du groupe. Entre négociation et délibération, ils se créent des marges de manœuvre afin de garantir leur pouvoir actionnarial et salarial.

\section{Choisir le repreneur}

Les deux offres (BNP et POP) sont déposées le 21 juin 2010. Les instances de représentation des salariés doivent s'exprimer sur chacune. Comme on l'a vu, il existe deux types d'instances qui sont « représentatives » des salariés du groupe, c'est-à-dire « considérées comme légitimes à agir au nom des salariés ${ }^{35}$, en tant qu'ils sont salariés (comité d'entreprise) mais aussi actionnaires (sociétés de personnels). Cette multiplicité d'instances de représentation permet d'étendre les «capacités politiques » des

35. Antoine Bevort, « Représentativité », in Antoine Bevort, Annette Jobert, Michel Lallement et Arnaud MiAs (dir.), Dictionnaire du travail, Paris, PUF, 2012, p. 666672. 
salariés en permettant une double audition des candidats à la reprise. Cela incite les candidats à expliciter de nombreuses facettes de leur offre.

«Les questionnements [...] sont aussi complémentaires. On voit bien : quand on est dans une AG de la SRM, c'est essentiellement des questions qui ont trait à l'éditorial, l'indépendance, les prises de contrôle, etc. [...] Parallèlement, le comité d'entreprise a aussi à prendre en charge toutes les questions d'organisation du groupe. » (Entretien avec un élu du comité d'entreprise de la SEM)

Tout d'abord, les sociétés de personnels doivent s'exprimer via leurs huit voix au conseil de surveillance de LMPA. En effet, les vingt membres du conseil de surveillance de LMPA sont appelés à se prononcer sur l'entrée en négociation exclusive avec l'une des deux offres, le 29 juin, pour trois mois, en échange d'une avance de trésorerie de 10 millions d'euros ${ }^{36}$. Pour cela, les sociétés de personnels du groupe auditionnent les deux trios d'investisseurs.

En parallèle, s'ouvre le 18 juin la procédure d'information-consultation des comités d'entreprise des différentes entités du groupe (Le Monde, Le Monde Interactif, Télérama, Courrier International, etc.). Les comités d'entreprise auditionnent donc également les candidats à la reprise (au titre de la représentation des salariés), le même jour. Par l'intermédiaire des questions écrites du comité d'entreprise posées aux repreneurs, les représentants des salariés demandent aux deux trios de s'expliquer sur 4 thèmes : finance et stratégie (13 questions), social (10 questions), gouvernance et structure (6 questions), éditorial (6 questions).

Ces consultations se soldent par un plébiscite massif de l'ensemble des personnels, appelés à choisir entre les offres le 25 juin, en faveur de l'offre BNP (tableau 2).

Tableau 2 : Part des personnels ayant voté pour l'offre BNP

\begin{tabular}{l|l} 
Journalistes du Monde & $90 \%$
\end{tabular}

Cadres du Monde $95 \%$

Employés du Monde $100 \%$

Le Monde Interactif $82 \%$

Les publications vie catholique

(magazines) $93 \%$

Courrier International $82 \%$

36. Pour éviter que le groupe ne se retrouve en cessation de paiement le 10 juillet. 
Comment expliquer ce choix massif des salariés envers l'offre BNP ? D'après l'expert mandaté par le comité d'entreprise, il y a eu un mélange entre des éléments d' «affect» et des éléments « rationnels» (entretien). Pour tenter de comprendre les raisons du choix des salariés, il nous faut étudier la manière dont les deux trios cherchent à convaincre les salariés du groupe de la justesse de leur offre sur les plans financiers et éditoriaux, à partir des offres écrites auxquelles nous avons eu accès, et des récits des présentations qu'ils en ont faits, aux sociétés de personnels et aux comités d'entreprise (questions écrites et audition).

L'offre POP déploie une rhétorique paternaliste qui se veut rassurante pour les salariés, mais laisse ouverte, comme on le verra, la discussion sur l'emploi. Le trio affirme avoir construit « un partenariat professionnel, stable et puissant» et s'engage à diriger le groupe « en bon père de famille, de manière prudente et raisonnable ». Les deux hommes de presse, déjà présents au capital du groupe (Perdriel au Nouvel Observateur et Juan Luis Cebrian pour Prisa) font état de leur «solidarité » en s'engageant à acquérir conjointement $70 \%$ de LMSA (via une société nouvelle détenue à parité entre eux, NewCo). Pourtant, leur prestation lors de la présentation des offres devant les sociétés de personnels est loin de démontrer cette unité, comme le prouve cet exemple :

« Perdriel et Prisa qui étaient ensemble n'étaient pas d'accord pour savoir si on produirait le journal le matin ou le soir ! » (Entretien avec un cadre du groupe après le vote des salariés)

L'offre BNP semble plus crédible et la présentation devant les personnels mieux réussie.

« Ce qui nous a fait pencher, [...] c'est surtout un côté contrat de confiance [...] Mais ça c'est de l'ordre du pari... [...] Il y a quelque chose qui s'est passé entre BNP et les personnels, ce sont des gens qui ont été convaincants. » (Entretien avec un journaliste après le vote des salariés)

Dans leur proposition, le trio BNP emploie davantage une rhétorique d'ordre sentimentale, évoquant « leurs sensibilités », « leurs cultures » et mettant en avant «l'histoire et les valeurs du groupe » Le Monde qui est «un bien commun». Ils se posent ainsi comme de riches mécènes qui veulent sauver le journal, et non comme des hommes d'affaires.

« Niel dit lui-même qu'il est scandaleusement riche! Quand on leur pose la question, ils disent qu'ils veulent défendre l'indépendance de la presse. » (Entretien avec un journaliste)

Le trio BNP propose la création d'une nouvelle société, Le Monde Libre (LML), dont chaque investisseur disposerait du tiers du capital so- 
cial et des droits de vote. Contrairement à l'offre POP qui commence sur l'exposition du projet financier et de la stratégie industrielle, le trio BNP ouvre son projet sur ses «engagements sur l'indépendance des rédactions » et sur la «minorité de blocage rendue au pôle d'indépendance ». Au contraire, ces questions sont reléguées dans l'annexe 1 de l'offre POP, qui détaille les «modalités spécifiques en faveur du pôle d'indépendance » à travers trois « piliers » : un comité des sages garant de l'indépendance des rédactions, une charte éditoriale, des droits de liquidité et de préemption. L'offre BNP va plus loin en proposant de restituer au pôle d'indépendance une minorité de blocage par un processus de relution. En clair, si les salariés ne sont plus le premier actionnaire et sortent du modèle co-gestionnaire, ils restent un actionnaire de référence via le pôle d'indépendance. C'est la proposition qui a séduit le conseil de gérance de la SRM :

« Ils se sont inscrits clairement dans la perspective d'accorder aux actionnaires historiques des droits spécifiques sanctuarisés et une place reconnue dans la gouvernance future du groupe. » (Extrait du communiqué de la SRM appelant à voter pour l'offre BNP, publié le jour du vote.)

Côté social, le projet POP prévoit une « clause de cession ${ }^{37}$ étendue à l'ensemble du personnel », formule qui masque un plan de départs volontaires (de 110 à 120 départs). Les salariés estiment avoir déjà « lourdement payé [leur] tribut ${ }^{38}$ » lors du plan de sauvegarde de l'emploi de 2008, qui a vu le départ de 110 salariés ${ }^{39}$. Au contraire, le trio BNP souhaite conserver ses effectifs, et espère qu'un minimum de journalistes se saisiront de la clause de cession pour quitter le groupe : « un plan social serait à nos yeux simplement destructeur de valeur ${ }^{40} \gg$. Face à une situation où l'emploi a été un temps la variable d'ajustement des salariés actionnaires, c'est ce point qui explique le choix des syndicats et des comités d'entreprise de se porter sur l'offre BNP.

«Les élus du comité d'entreprise et de l'intersyndicale se sont ralliés à cette solution [BNP] présentant [...] le moins de risques sur le plan social. » (Extrait de

37. La clause de cession est nécessairement ouverte lors d'un changement de propriétaire. Elle permet aux journalistes qui le souhaitent de quitter l'entreprise tout en bénéficiant d'indemnités de licenciement.

38. Extrait de l'avis du comité d'entreprise de la SEM sur le projet de recapitalisation rendu le 14 septembre 2010.

39. Pour une histoire du PSE, cf. Camille DUPUY, «L'entreprise de presse en conflit. Libération et Le Monde en restructuration », Travail et Emploi, $\mathrm{n}^{\circ}$ 124, 2011, p. 29-42.

40. Extrait des réponses écrites des candidats à la reprise aux questions du comité d'entreprise (24 juin 2010) 
l'avis du comité d'entreprise sur le projet de recapitalisation remis le 14 septembre 2010)

Ainsi, au-delà de ces différences rhétoriques (paternaliste pour POP ou sentimentale pour BNP), le choix massif des salariés pour l'offre BNP s'explique par deux éléments qui ont su convaincre les deux formes de représentation : l'indépendance pour la première (société de personnels) et les conditions sociales pour la seconde (comités d'entreprise). Tout en ménageant une place à l'héritage de J. Schwœbel, (mais en le cantonnant à un «pôle d'indépendance » nettement minoritaire), les propositions du trio BNP apportent des garanties satisfaisantes sur le terrain de l'emploi, qui avait été délaissé par les salariés actionnaires, et qui suscite une adhésion de l'ensemble des salariés (au-delà des seuls journalistes). Les aspects liés à la « gouvernance » sont prioritaires dans le choix des salariés, en réservant la question des choix éditoriaux pour un second temps. Les négociations s'ouvrent donc dans un climat serein avec ces deux formes d'instances.

\section{Maintenir l'héritage : le pôle d'indépendance et la charte d'éthique}

L'enjeu de la négociation exclusive pour les sociétés de personnels est de garantir leur indépendance, par la conservation de certains droits liés à leur pouvoir actionnarial, malgré l'horizon de dilution de leur droit de propriété à la fin du processus. Le conseil de surveillance de LMPA doit se prononcer entre les deux offres pour entériner l'entrée en négociation exclusive. Comme on l'a vu, la SRM ne dispose pas de droit de veto sur cette opération, qui requiert la majorité simple au sein du conseil (10 voix sur 20, tableau 1). Les sociétés de personnels ne disposant que de 8 sièges sur 20 peuvent théoriquement être mises en minorité ce qui signifierait une entrée en négociation exclusive avec l'offre POP. Et, de fait, les 12 autres voix sont favorables au trio POP.

Cependant, si la SRM ne dispose pas à elle seule d'un droit de veto légal, les sociétés de personnels disposent d'un droit de veto moral, conforté par la consultation des salariés.

«Le vote était tellement massif de la part des sociétés de personnels que politiquement ça n'était pas possible. Ça aurait été vu comme les capitalistes [actionnaires externes] du groupe privilégient la solution Sarkozy ${ }^{41}$, ça tuait le journal... Mais juridiquement c'était possible. » (Entretien avec le président de la SRM)

41. Alors président de la République, qui s'était prononcé en faveur de l'offre POP. 
Le trio POP retire son offre au début du conseil de surveillance, ne souhaitant pas «passer en force » contre l'avis des salariés. La question qui est posée aux membres du conseil de surveillance devient donc celle d'accepter ou de refuser l'offre BNP. L'offre est acceptée par 10 membres : les 8 voix des sociétés de personnels, la voix du président du conseil (Louis Schweitzer) et la voix de Claude Perdriel lui-même. Ne souhaitant pas s'opposer au choix des personnels (« vote de solidarité »), tout en ne voulant pas plébisciter l'offre BNP («vote de vigilance »), les 10 autres membres s'abstiennent.

Tout en préservant une certaine indépendance à l'égard du pouvoir «de l'argent », mais aussi dans la grande tradition de la profession journalistique, à l'égard du pouvoir politique, le vote au sein de LMPA traduit l'influence des salariés dans leur ensemble, qui s'est manifestée par une procédure de vote des salariés. On constate ainsi une forme de transition dans le pouvoir d'influence des représentants des salariés, passant d'un pouvoir actionnarial à un pouvoir plus directement salarial.

Ce vote ouvre une période de négociation exclusive avec l'offre BNP. Les sociétés de personnels négocient les conditions de leur perte de droit actionnarial, avant d'entériner ou non la prise de contrôle des actionnaires à l'issue des trois mois, par un nouveau vote, à la majorité qualifiée cette fois (14 voix sur 20). Les représentants des salariés agitent la menace de ne pas entériner la prise de contrôle des actionnaires, afin de contraindre les investisseurs à négocier avec eux cette perte de contrôle.

«Un certain nombre de points devront notamment être précisés. [...] Le conseil et avec lui tout le pôle d'indépendance considère comme préférable que la perte de contrôle ne soit entérinée qu'à l'issue de ce processus. » (Communiqué du conseil de gérance de la SRM, le 25 juin 2010)

On voit, ici encore, comment les salariés utilisent les règles comme des armes dans la négociation. Cependant, on peut s'interroger sur la crédibilité de cette menace puisque le refus d'entériner la prise de contrôle conduirait le groupe Le Monde à devoir rembourser les 10 millions d'euros avancés par le trio, à un taux d'intérêt très élevé (17\%). Les investisseurs disposent donc eux aussi d'un atout pour modérer les appétences de la SRM qui ne peut pas vraiment se permettre de faire échouer le processus.

Chaque partie disposant d'une arme (juridique ou financière) forte, les négociations entre les actionnaires et le pôle d'indépendance se déroulent dans une situation de pouvoir relativement équilibrée. Elles portent sur deux points : la minorité de blocage du pôle (conserver un pouvoir actionnarial) et la rédaction d'une charte assortie de comités de contrôle. 
Le pôle d'indépendance est constitué des associés salariés (sociétés de personnels) et des associés historiques (sociétés de lecteurs et personnalités indépendantes qui remplacent l'association Hubert Beuve-Méry). Au terme de la recapitalisation, ce pôle qui était anciennement actionnaire majoritaire de LMSA (via LMPA) aurait perdu tout pouvoir de contrôle, au profit de LML. Le compromis, envisagé par Pierre Bergé, est la création d'une société en commandite simple (SCS) dont le commanditaire est la société de Pierre Bergé, et le commandité la SARL du pôle d'indépendance, qui reçoit 10 millions d'euros, permettant d'accéder à une minorité de blocage $(33,34 \%)$ dans LMSA. Cette structure permet de dissocier les droits politiques du pôle de ses droits économiques (les éventuels dividendes seraient versés à la SCS de Pierre Bergé). Le pôle dispose de plusieurs droits : droit de préemption (priorité en cas de vente de parts), droit d'anti-dilution (sa part doit rester la même dans LMSA), droit de veto sur le transfert de la société éditrice du Monde ou d'une filiale. Le pôle bénéficie de 5 sièges sur 18 au nouveau conseil de surveillance de LMSA $^{42}$. La création du pôle d'indépendance permet donc aux salariés de se faire reconnaître de nouveaux droits d'actionnaires par la négociation, droits qui ne leur étaient pas dus, mais acceptés par les nouveaux actionnaires pour préserver un «climat social apaisé » (entretien). Cependant, ils bénéficient de nettement moins de pouvoirs liés à l'actionnariat qu'avant la recapitalisation.

«Je ne vais pas vous dire qu'on y a gagné, mais en jouant le jeu de la collectivité des salariés et des lecteurs, on a réussi quand même à garder un poids non négligeable au sein du groupe, et même la possibilité un jour de reprendre le contrôle du groupe. » (Entretien avec le président de la SRM)

Le second verrou statutaire qui est discuté avec les personnels au cours de la période de négociation exclusive est la rédaction d'une charte, publiée la première fois le 3 novembre 2010, puis régulièrement dans l'ensemble des titres. Elle a pour objet de « rappeler les principes essentiels d'indépendance $[\ldots]$ et de préciser les droits et devoirs des journalistes, des dirigeants comme des actionnaires ${ }^{43} \gg$. Elle est inscrite dans les statuts, ce qui la rend opposable. Sont donc créées des structures pour recevoir et traiter les plaintes, sous la forme de deux comités d'éthique et de déontologie (un pour Le Monde et ses suppléments, déclinaisons, sites numériques ; un pour les magazines). Les comités disposent en outre d'un droit de veto sur le développement d'activités qui utiliseraient la marque Le

42. D'après le protocole d'accord signé entre LML et le pôle d'indépendance, le 2 novembre 2010.

43. Extrait de la charte d'éthique et de déontologie du groupe Le Monde. 
Monde et pourraient objectivement lui porter atteinte. Au-delà de leur pouvoir politique d'actionnaire, les salariés s'intéressent donc aussi à ce que va devenir leur produit (ce qui n'était pas le cas au moment du choix des offres).

Les sociétés de personnels réussissent à imposer des verrous statutaires dans l'espoir de garantir leur indépendance, en faisant planer la menace de ne pas entériner le processus de recapitalisation. Ils conservent ainsi une part de leur pouvoir d'actionnaire.

\section{Négocier les conditions du rachat : l'information-consultation du comité d'entreprise}

Parallèlement, se déroulent les procédures d'information-consultation des onze comités d'entreprise des titres du groupe, par lesquels s'exprime le pouvoir salarial, conféré aux salariés par le droit du travail. C'est une procédure légale qui impose à la direction de consulter les élus des comités d'entreprise afin que ceux-ci rendent un avis permettant d'entériner la prise de contrôle en conseil de surveillance ${ }^{44}$. Ce cadre légal permet, dans le cas de transferts d'entreprise, d'engager un véritable débat sur l'avenir de l'entreprise et de son personnel. La question posée est celle de « la recapitalisation du groupe, telle qu'elle va être proposée par un investisseur [encore inconnu au début du processus], avec ses impacts juridiques et financiers ${ }^{45} »$.

La procédure a pour objectif de permettre aux comités d'entreprise de rendre un avis «éclairé » sur les évolutions que proposent les futurs dirigeants. Elle ouvre une marge de manœuvre beaucoup plus crédible que celle d'un refus éventuel de la recapitalisation (modalité du pouvoir actionnarial revendiqué par la SRM) en conférant aux comités d'entreprise la possibilité de ne pas rendre d'avis et, ainsi, de bloquer la procédure.

«C'était la hantise de nos directions, qu'un comité d'entreprise ne rende pas d'avis, auquel cas il aurait fallu engager une procédure judiciaire pour obliger le comité d'entreprise à rendre un avis. On aurait pu bloquer la procédure. » (Entretien avec un élu du comité d'entreprise de la SEM)

Les élus aux comités d'entreprise utilisent cette marge de manœuvre pour desserrer les contraintes imposées par la direction. En effet, au départ, il leur est demandé de rendre un avis très rapidement, avant le 9 juillet 2010 (veille du jour où le journal se trouverait en cessation de paie-

44. En vertu des articles L 2323-6 et 2323-19 du code du travail.

45. Selon le DRH de la SEM, extrait du PV de comité d'entreprise de la SEM du 18 juin 2010 . 
ment). C'est la raison pour laquelle la procédure d'information-consultation s'ouvre le 18 juin ( 3 semaines avant), avant même l'entrée en négociation exclusive (28 juin). David Guiraud parle ainsi de «la nécessité d'anticiper par cette réunion zéro afin de débattre, de gagner du temps, de tenir les échéances ${ }^{46} »$. Au contraire, les salariés souhaitent plus de temps et sortir d'une procédure « à la hussarde », « à la va-vite » ${ }^{47}$. La date de la remise des avis est reportée le plus tard possible, au 14 septembre (afin de disposer des deux semaines nécessaires pour convoquer le conseil de surveillance le 28 septembre, veille de la fin de la période de négociation exclusive).

Les procès-verbaux des 5 réunions du comité d'entreprise de la SEM ( 2 avant la négociation exclusive et 3 après) qui travaille en intersyndicale (SNJ, CGT, CFDT) nous ont permis, à partir du cas de ce comité d'entreprise, d'interroger la place des différents acteurs (salariés, direction et actionnaires) dans la négociation. Les réunions du comité d'entreprise de la SEM peuvent être réparties en deux types : 3 d'entre elles (18 juin, 29 juin, 14 septembre) mettent en présence les salariés et leur direction. Elles sont courtes (moins d'une heure) et informatives (procédure, échéance, rendu de l'avis). Il est intéressant de noter qu'Éric Fottorino, président du directoire du Monde (et donc du comité d'entreprise) en est absent pour deux d'entre elles. Il semble que la direction soit très en retrait dans ce processus, comme l'avoue Éric Fottorino : «vous devez savoir que David Guiraud et moi-même ne sommes pas toujours au cœur des discussions $\gg$ confie-t-il aux salariés ${ }^{48}$. C'est pourtant la direction qui est censée porter le projet des actionnaires. "Qui seront nos interlocuteurs ?» s'interroge alors un élu du comité d'entreprise ${ }^{49}$. Les vrais interlocuteurs sont les actionnaires (ou leurs représentants), comme le prouvent les deux autres réunions qui se tiennent au cours de la procédure d'information-consultation ( 24 juin et 2 juillet ${ }^{50}$ ) qui les invitent à expliciter leur projet (de même avec les 10 autres comités d'entreprise). Pourtant, les actionnaires éventuels semblent mal à l'aise avec cette position : « je ne sais même pas à quel titre nous vous parlons aujourd'hui » expliquent-ils ${ }^{51}$.

46. Extrait du PV du comité d'entreprise de la SEM du 18 juin 2010.

47. Selon un élu syndical, extrait du PV du comité d'entreprise de la SEM 29 juin 2011.

48. Extrait du PV du comité d'entreprise de la SEM du 18 juin 2010.

49. Ibidem.

50. Avec Xavier Niel et Louis Deryfus, conseiller de Mathieu Pigasse.

51. Xavier Niel, extrait du PV du comité d'entreprise de la SEM, 2 juillet 2010. 
Comme pour l'audition avant les votes, la double représentation des salariés (sociétés de personnels et instance de représentation des personnels salariés) au cours de cette période leur permet d'interroger doublement les investisseurs. Lorsqu'on liste les questions posées par les membres du comité d'entreprise de la SEM aux repreneurs, on s'aperçoit qu'elles rejoignent les interrogations propres au pouvoir actionnarial, portant sur le maintien d'un contrôle de la structure capitalistique. C'est le sens de la remarque de David Guiraud : "vous allez vous prononcer sur un projet global dans lequel figurera un certain nombre de critères d'appréciation, que vous connaissez par cœur, comme l'indépendance, la solidité financière, la gouvernance ${ }^{52}$ ». Mais, dans ce processus, le comité d'entreprise découvre pleinement ses prérogatives, en envisageant les volets sociaux et économiques de l'opération (plan de départs, maintien des accords collectifs, remplacement des départs en clause de cession, salaires et primes, embauche des précaires, sous-traitance). Cela rejoint les initiatives des différents comités d'entreprise qui, tous, ont mandaté des experts économiques pour leur permettre de rendre un avis « éclairé ». Trois cabinets sont mandatés par les 11 comités d'entreprise. Le cabinet mandaté par la SEM a rendu son rapport le $1^{\text {er }}$ septembre 2010, dressant un état des lieux de la situation, et pointant les «points forts » (crédibilité financière, profils des investisseurs, garanties d'indépendance, droits spécifiques conférés au pôle d'indépendance) et des «points faibles » (des projets industriel et financier peu détaillés, des perspectives d'emploi non sanctuarisées, la question de la sortie des actionnaires minoritaires qui n'est pas réglée). Ici encore, on voit que les thèmes abordés vont au-delà de la question du contrôle capitalistique vu par l'expert comme « une particularité au journal Le Monde »(entretien). Tout en retrouvant la main sur les questions sociales et financières, les élus du comité d'entreprise s'intéressent également à l'avenir de l'entreprise et de son produit (indépendance, ligne rédactionnelle), dans le cadre de débats évoquant le registre transactionnel mis au jour dans les restructurations à Canal Plus ${ }^{53}$, à Libération et au Monde ${ }^{54}$. Cela s'explique par le fait que, dans ces entreprises, s'engage une éthique professionnelle au sein des relations de travail, qui pousse les comités d'entreprise à s'inscrire dans un registre délibératif sur l'avenir de l'entreprise.

52. Extrait du PV du comité d'entreprise de la SEM, 18 juin 2010.

53. Claude DIDRY, «De la restructuration dans l'entreprise à la restructuration des relations professionnelles : le cas de Canal Plus », L'homme et la société, n 63-64, 2007, p. 95-124.

54. Camille DupuY, «L'entreprise de presse en conflit. Libération et Le Monde en restructuration »,op. cit. 
Le comité d'entreprise de la SEM rend le 14 septembre un avis «en faveur de la solution formulée par MM. Bergé, Niel et Pigasse [...] assorti de réserves » (sur des questions sociales et stratégiques). Les 10 autres comités d'entreprise ont suivi une procédure similaire, comme par exemple au Monde Interactif.

\footnotetext{
«J'ai fait le PV de comité d'entreprise le plus long de l'histoire de l'entreprise ! On a ouvert un comité d'entreprise qui s'est refermé 4 mois plus tard, après 7 ou 8 suspensions de séance, deux auditions, des présentations de documents, une rencontre avec Louis Dreyfus, tout un tas de trucs! On a finalement rendu un avis qui n'était ni positif, ni négatif, puisqu'on a découvert [...] qu'on devait juste rendre un avis (qui ne devait pas être blanc ou noir). » (Entretien avec un élu du comité d'entreprise de MIA)
}

Ainsi, jusque dans cette procédure de la négociation exclusive, les salariés, par l'intermédiaire des sociétés de personnels actionnaires et par les comités d'entreprise, se placent au centre des discussions avec les repreneurs, tout en marginalisant la direction. Il se dessine une transformation progressive du mode d'intervention des salariés, avec, d'une part, l'affaiblissement du pouvoir actionnarial et, d'autre part, le renforcement du pouvoir salarial.

\section{III - Être salarié dans le nouveau groupe Le Monde}

La vente du groupe marginalise le pouvoir actionnarial. Dans ce nouveau cadre, les salariés découvrent l'exercice d'un nouveau pouvoir salarial. Plusieurs épreuves marquent cet apprentissage. Cela permet aux instances représentatives du personnel de se saisir de leurs nouveaux droits et de développer leur capacité à les utiliser.

\section{«Écrire une nouvelle page »}

Le conseil de surveillance de LMPA entérine l'arrivée du trio BNP dans l'actionnariat du groupe au terme de la réunion du 29 septembre 2010. Le processus est finalisé (closing) le 2 novembre. Au terme de cette recapitalisation, les actionnaires internes, à savoir les salariés du groupe, et les rédacteurs du Monde en particulier, ont perdu leur place de premier actionnaire. La structure juridique qui était censée se simplifier se complexifie (figure 3). La société éditrice du Monde, structure porteuse des filiales, est désormais détenue à $55 \%$ par Le Monde Libre (LML de Bergé, Niel et Pigasse, auquel Prisa est venu se greffer) et à 33,34\% par le pôle d'indépendance (qui en détient $17 \%$ au titre de sa participation dans LMPA, auquel s'ajoute les 16,34\% issus de la SCS de Pierre Bergé). Les parts restantes se distribuent entre plusieurs petits actionnaires. 
Le centre du pouvoir qui se trouvait dans LMPA descend donc au niveau de LMSA. La structure du conseil de surveillance du groupe reflète la main mise de LML sur les pouvoirs de décision : sur les 18 sièges, 10 sont attribués à LML, 5 au pôle d'indépendance, 1 aux actionnaires minoritaires et 1 au groupe Prisa (en négociation pour entrer dans LML). Ainsi :

«Recapitalisation est un doux euphémisme pour parler de prise de contrôle. » (Entretien avec un délégué syndical du Monde)

Figure 3 : Structure du groupe après la recapitalisation

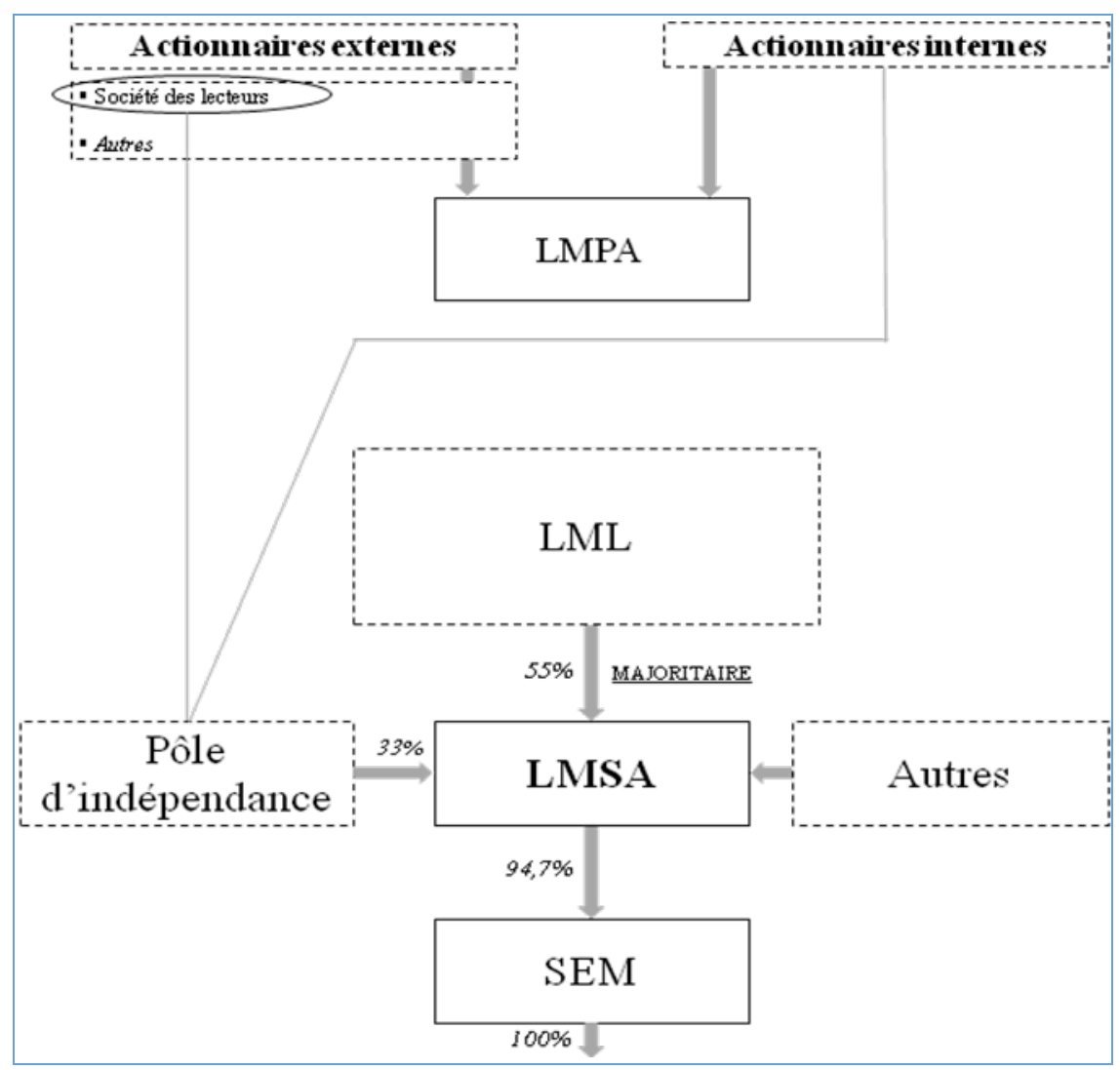

Cette perte de contrôle des sociétés de personnels constitue un changement radical dans l'histoire du journal et de son groupe. Au lendemain de la recapitalisation, le directeur du groupe, Éric Fottorino estime que « ces changements profonds de structure ouvrent à l'évidence une ère 
nouvelle ${ }^{55} »$. Cette rhétorique du changement et de la rupture se retrouve tout au long de nos entretiens menés après le mois de novembre 2010. Le président de la société des rédacteurs du Monde parle d'un «paradigme complètement nouveau » et d'une «nouvelle culture d'entreprise». Du côté des actionnaires, sont évoqués un «moment charnière » et un «changement d'époque ». Pour résumer :

« On change de paysage, de patron, d'époque.» (Entretien avec un délégué syndical du Monde)

Alors qu'Éric Fottorino salue début novembre « la recapitalisation réussie de notre maison », les salariés s'inquiètent de cette prise de pouvoir capitalistique par les nouveaux arrivants : " on donne les clefs de la maison » s'exclame le président de la société des rédacteurs (entretien). Ce registre des «clefs » sera largement repris, comme en témoigne un dessin de Plantu intitulé «Les Clefs du Monde ${ }^{56} »$. Cette inquiétude fait apparaître une vision patrimoniale de l'entreprise à laquelle un certain nombre de salariés renoncent difficilement.

L'effacement de la direction, nommée par les sociétés de personnels (les actionnaires internes disposaient d'un droit de veto sur leur directeur, qu'ils ont perdu lors de la recapitalisation), s'accompagne d'un glissement sémantique à propos de l'arrivée de nouveaux actionnaires, qui est vécue comme l'arrivée de patrons. Ainsi, selon un délégué syndical, «Maintenant, on a des vrais patrons » (entretien). L'installation du trio BNP dans l'actionnariat du groupe aboutit ainsi à une véritable expérience patronale, avec le remplacement des « dirigeants » issus des sociétés de personnels par Louis Dreyfus, le conseiller d'un des membres du trio, qualifié alors de «patron ». Pourtant, au cours de la réunion du comité d'entreprise du 2 juillet, Niel avait bien affirmé : « il n'est pas question de nommer [Dreyfus] à un poste quelconque dans le groupe ». Cette expérience constitue une véritable épreuve, montrant les limites des verrous statutaires négociés au cours du processus de reprise. Les salariés décrivent, après le mois de décembre 2010, «une mauvaise ambiance » empreinte d' « incertitudes », d' ' angoisses », de « morosité » (extraits d'entretiens). «On sait où est le pouvoir, on ne sait pas encore comment va s'organiser le contre-pouvoir » raconte un salarié du groupe (entretien). À travers l'étude des structures et des négociations postérieures à la recapitalisation, nous allons essayer de déterminer si les salariés disposent toujours de pouvoirs dans cette entreprise et leur nature. Quelle est la capa-

55. «Écrire une nouvelle page », Le Monde du 3 novembre 2011.

56. Paru dans Le Monde Magazine, $\mathrm{n}^{\circ} 55$ (octobre 2010). 
cité des salariés à mobiliser leurs nouveaux droits et à les transformer en pouvoirs, sachant que l'existence d'un droit ne garantit pas nécessairement la possibilité d'exercice de ce droit. Pour cela, nous allons étudier les deux forces salariales que sont le pôle d'indépendance (du côté du pouvoir actionnarial) et les instances de représentation du personnel $(\mathrm{du}$ côté du pouvoir salarial).

\section{Les nouvelles limites du pouvoir actionnarial}

Les sociétés de personnels ont négocié qu'une partie de leur pouvoir actionnarial leur soit restituée dans une structure qui les agrège. Le protocole d'accord du pôle d'indépendance (PAPI) est signé par les sociétés de personnels et LML le 2 novembre 2010. Le pôle se répartit entre deux collèges : les associés salariés et les associés historiques (encadré 3), et dispose de 5 sièges sur les 18 du conseil de surveillance de la SEM et du groupe. La création de ce pôle est le premier pas vers la création d'un groupe cohérent.

\footnotetext{
«Finalement, ce groupe vient de naître. Car on a fait tout un travail en commun sur la recapitalisation. [...] On a pris des décisions ensemble, à des majorités plus écrasantes les unes que les autres. C'est la première page qu'on écrit ensemble, parce que jusque-là, on a écrit une page sur leur dos... » (Entretien avec un salarié du Monde)
}

On l'a vu, des droits particuliers sont conférés au pôle d'indépendance : droit de veto sur le transfert du quotidien, d'une de ses déclinaisons ou d'un magazine; droit de préemption sur toute cession d'actions ; droit d'information sur toute prise de position (même indirecte) procurant à un tiers plus de $25 \%$ des droits de vote de LML. Pour exercer ces droits, la majorité (simple ou des $2 / 3$ selon les questions) ainsi que l'assentiment d'au moins un membre de chaque collège sont requis. Cependant, il se pose la question de la capacité du pôle à utiliser ces droits, notamment dans le cas du droit de préemption, dans la mesure où instaurer une priorité aux salariés à la reprise des actions suppose qu'ils disposent du capital nécessaire au rachat. De même, le fait que «le droit de veto doit être exercé de bonne foi et ne pas empêcher la réalisation d'une opération que l'intérêt du groupe commanderait ${ }^{57} \gg$ limite considérablement le pouvoir du pôle en cas de besoins financiers. Si la société des rédacteurs du Monde voit ses pouvoirs diminuer, certains titres au contraire ont acquis des avantages via le pôle d'indépendance dont ils ne disposaient pas avant. Par exemple, tous les titres disposent désormais d'un droit de veto sur la

57. Extrait du protocole d'accord. 
nomination de son directeur, qui doit être approuvée par $50 \%$ des journalistes en CDI dans les magazines, $60 \%$ pour le quotidien. Ce droit existait déjà au Monde et à Télérama, mais l'alignement des avantages sur l'ensemble du groupe a permis à Courrier International, par exemple, d'en bénéficier.

«On a gagné le droit de veto sur la vente. On a gagné un vote sur le directeur de la rédaction.» (Entretien avec le président de la société des personnels de Courrier International)

Les membres de la SRM constatent une diminution de leur pouvoir actionnarial. Il semble que nous puissions nuancer cette conclusion à plusieurs égards. Tout d'abord, la SRM avait déjà vu son pouvoir décroître dans la mesure où il lui était difficile d'exercer ses droits d'actionnaire avant la recapitalisation. En effet, « le droit le plus visible qui lui restait c'était le droit de veto sur le président du directoire du groupe » se souvient le président de la SRM (entretien), pourtant, il lui a été coûteux de l'exercer en 2008 : le veto de la SRM sur le renouvellement du mandat de Jean-Marie Colombani et la pression qu'elle a exercée pour pousser Alain Minc à démissionner lui ont coûté la démission forcée de son président, Jean-Michel Dumay.

La seconde nuance que nous pouvons apporter quant à la diminution du rôle de la SRM tient à la large place qu'elle s'octroie via le pôle d'indépendance (tableau 3). La répartition des sièges au sein du pôle montre une nette prépondérance des personnels du Monde sur le reste du groupe (6 sur 11$)$.

Les personnels du Monde représentent pourtant à peine plus du tiers des salariés du groupe $\left(477\right.$ sur $\left.1248^{58}\right)$. Parmi ces personnels, les journalistes du Monde (278 soit un peu plus de $20 \%$ des salariés du groupe) capitalisent à eux seuls 3 des 11 voix $(27 \%)$ distribuées entre les associés salariés. «Le nombre de voix est pondéré en fonction de l'importance de la participation de chacun dans le capital du groupe » explique un journaliste du Monde (entretien). Ainsi, tout en ayant perdu de son pouvoir, la SRM réussi à conserver sa domination sur les affaires du groupe.

«La SRM a bien œuvré pour garder du pouvoir un peu partout, puisque son président est aussi président du pôle d'indépendance, vice-président de LMSA, a un siège dans le comité d'éthique... » (Entretien avec un cadre du groupe)

58. Ces chiffres correspondent à la situation au 31 décembre 2009. 
Tableau 3 : Répartition des sièges au sein du pôle d'indépendance

\begin{tabular}{|c|c|}
\hline Associés salariés & 11 \\
\hline Le Monde & 6 \\
Société des rédacteurs du Monde & 3 \\
Société des personnels du Monde & 1 \\
Société des employés du Monde & 1 \\
Société des cadres du Monde & 1 \\
Autre titres & 3 \\
Société des personnels des publications vie catholique & 1 \\
Société des personnels de Courrier International & 1 \\
Société des personnels du Monde Interactif & 1 \\
\hline Associés historiques & 4 \\
\hline Sociétés des lecteurs & 2 \\
Personnalités indépendantes & 2 \\
\hline
\end{tabular}

Nous avons pu évaluer les capacités de la SRM à exercer ses droits lors de la nomination du nouveau directeur de la rédaction (février 2011), suite au limogeage d'Éric Fottorino (décembre 2010). Une commission tri-partite est créée, composée d'un actionnaire (Pierre Bergé), du directeur du groupe (Louis Dreyfus) et du président de la SRM (Gilles Van Kote). La SRM souhaite que la commission constitue une short list de trois candidats entre lesquels l'assemblée générale des journalistes puisse choisir. Bergé et Dreyfus s'y opposent et ne présentent au vote de la SRM qu'un seul candidat : Erik Izraelewicz. La SRM n'utilise pourtant pas son droit de veto, risquant de faire obstacle à la nomination d'un directeur dont le journal a besoin. Ils sont donc contraints d'accepter cette candidature. Ainsi, si le droit de nommer le directeur du journal est conservé, son exercice est compliqué, traduisant une perte du pouvoir actionnarial de la SRM.

«On se rend compte que la SRM a un pouvoir très limité. On n'a pas utilisé le droit de veto, ça a voté à $80 \%$ pour Izraelewicz, alors que le processus préconisé par la SRM sur le choix du futur directeur du Monde n'a pas été respecté.» (Entretien avec un délégué syndical du Monde) 
Ainsi, bien que le protocole d'accord établisse une «habilitation du chef d'entreprise par les salariés » sur le modèle proposé par certains chercheurs ${ }^{59}$, ce cas montre que l'établissement de ce droit est nécessaire mais pas suffisant pour que les salariés participent effectivement à la nomination de leur dirigeant. Au terme de cette épreuve de force, les membres de la SRM prennent conscience de la perte d'un pouvoir de contrôle et sont conduits à envisager un champ d'intervention nouveau qui se rapproche de celui des sociétés de journalistes classiques.

«Depuis ces dernières années, la SRM a consacré toutes ses forces aux questions économiques et de gestion et a négligé, par la force des choses, son rôle de société de journalistes, ce rôle sur le terrain éditorial, de la déontologie. C'est un terrain qu'on va réinvestir mécaniquement. [...] C'est une nouvelle histoire, un nouveau schéma, un nouveau fonctionnement de la SRM.» (Entretien avec le président de la SRM)

La SRM, acteur essentiel du pouvoir actionnarial comme garantie de l'indépendance, est donc conduite à se déplacer sur un nouveau terrain. Cette épreuve traduit son ralliement aux activités traditionnelles dévolues au pouvoir salarial (emploi, contenu), en se trouvant privée d'un droit de regard sur la gestion de l'entreprise. Le pouvoir salarial se redéploie dans un exercice par de nouveaux acteurs.

\section{Redécouvrir le pouvoir salarial : nouveaux acteurs, nouveaux conflits}

Face à la perte du pouvoir actionnarial, les salariés réactivent le droit du travail. Cela permet de sortir d'une vision de l'entreprise dans laquelle la responsabilité est uniquement attachée aux droits de propriété, pour envisager l'entreprise comme un espace de coopération au sein duquel les salariés ont également la compétence d'évaluer l'entreprise ${ }^{60}$. Au sein du groupe, les onze comités d'entreprise jouent un rôle important dans la recapitalisation, puisqu'ils doivent remettre un avis éclairé le 14 septembre sur l'offre des actionnaires. Les comités d'entreprise, et avec eux l'ensemble des instances représentatives du personnel, se sont toujours inscrits à côté des sociétés de personnels, ce qui explique notamment qu'ils auditionnent les candidats à la reprise (juin 2010) le même jour mais à l'occasion de deux réunions différentes. Les comités d'entreprise s'affirment par rapport aux sociétés de personnels, en interrogeant les deux trios

59. Cf. notamment Armand Hatchuel et Blanche SEGRESTin, Refonder l'entreprise, op. cit.

60. C'est l'une des propositions de Roger BAUDOIN (éd.), in L'entreprise : formes de la propriété et responsabilité sociale, op. cit. 
dans un sens spécifique, qui intègre un regard sur le devenir des personnels et une attention à la stratégie éditoriale proposée.

En somme, les deux instances s'informent de l'avancée de dossiers qu'elles ont respectivement en charge mais tentent de conserver leurs distinctions. «Chacun chez soi » raconte un délégué syndical. Il y a un « jeu de casquette ».

«Ça ne veut pas dire qu'on ne marchait pas de pair, en informel, et qu'on n'échangeait pas les avis, mais surtout pas de façade. Les choses étaient vraiment séparées. » (Entretien avec le président d'une société de personnels)

L'arrivée d'actionnaires capitalistes tranche les problèmes que posait jusqu'alors l'exercice d'un pouvoir actionnarial par les salariés, lié à l'existence de deux instances représentatives des salariés dont les intérêts pouvaient être divergents. En effet, la recapitalisation signe la fin de l'actionnariat salarié tel qu'il était conçu par le passé (majoritaire). Ce nouveau mode de gouvernance reconfigure les relations professionnelles, entendues comme les relations qui s'établissent entre les salariés et leurs représentants et les employeurs et leurs organisations dans l'entreprise et qui ont pour objet «l'élaboration de règles de contenu ${ }^{61}$ ». En effet, on assiste à une normalisation des relations professionnelles qui auparavant étaient confuses du fait de l'actionnariat salarié. Face à cette reconfiguration, les instances représentatives des salariés retrouvent une place prépondérante par l'expression collective d'un pouvoir salarial qui ne se heurte plus à un pouvoir actionnarial qui était également entre leurs mains. En se retrouvant face à des actionnaires «capitalistes » et à une direction choisie par ces derniers, les syndicats ouvrent une nouvelle ère dans la négociation salariale.

«On était gentil, les directions du Monde ont toujours été gentilles c'est-à-dire pas très combatives. » (Entretien avec un délégué syndical du Monde)

Les syndicats ont un nouveau rôle à jouer. Il doit s'établir un nouveau rapport de force entre salariés, directions et actionnaires.

« À nous de dire, de camper, de proposer, d'installer un rapport de force. On va installer un rapport de force, ce qui n'était pas complètement dans la culture. » (Entretien avec un délégué syndical du Monde)

Pour essayer de comprendre comment se reconfigurent les relations de pouvoir entre ces acteurs, nous avons étudié la négociation de la clause de cession, entre la direction du groupe et l'ensemble des délégués syndicaux. La loi prévoit qu'un journaliste peut démissionner pour l'une des raisons

61. Antoine BEVORT et Annette JOBERT, Sociologie du travail : les relations professionnelles, Paris, Armand Colin, 2011. 
suivantes : cession du journal, cessation de la publication du journal ou changement notable dans le caractère ou l'orientation du journal ${ }^{62}$. Dans ces cas, le salarié qui rompt le contrat de travail a le droit aux indemnités prévues lors d'un licenciement. Cela prouve le caractère moral (et non seulement financier) qui lie le salarié à son entreprise. Le contrat de travail engage donc plus que l'échange d'un service (rédaction d'articles) en contrepartie d'un salaire. C'est «l'honneur, la réputation ou, d'une manière générale, les intérêts moraux ${ }^{63} »$ qui sont en jeu dans le contrat de travail puisqu'il peut être rompu pour ces motifs. L'important changement dans l'actionnariat du groupe Le Monde déclenche automatiquement l'ouverture de la clause de cession pour les journalistes du groupe. Le financement de cette clause est prévu à hauteur de 10 millions d'euros dans les rapports des experts des comités d'entreprise.

L'accord signé le 13 décembre 2010 par les délégués syndicaux de l'ensemble des entités du groupe (Télérama, Culturama, Courrier International, Malesherbes Publications, Le Monde Interactif, LePost.fr, la SEM, Le Monde diplomatique) concernant les conditions d'application de la clause de cession n'est pas très favorable. Il prévoit que les indemnités versées en cas de départ soient de 1 mois par année d'ancienneté, ce qui est inférieur à ce qui avait été négocié lors des départs volontaires du dernier plan de sauvegarde de l'emploi (2008). Mais en contrepartie, une négociation s'ouvre sur l'emploi dès janvier 2011 (remplacement des départs et titularisation des précaires). Les délégués syndicaux ont donc commencé à établir ce rapport de force en échangeant leur signature contre des engagements en termes d'emploi. "D'une négociation sur les conditions de départ, c'est devenu une négociation sur l'emploi ». En effet, l'accord prend acte «que la clause de cession ne saurait être assimilée à un plan de sauvegarde de l'emploi ${ }^{64}$. Cette négociation a porté ses fruits puisque 60 journalistes ont été embauchés et 40 titularisés depuis la recapitalisation, contre 50 départs en clause de cession, soit un effectif net de +10 journalistes ${ }^{65}$. Les syndicats réinvestissent les questions d'emploi en transformant les enjeux de la négociation, utilisant leurs capacités politiques.

62. Article L. $7112-5$ du Code du travail.

63. Ibidem.

64. Extrait de l'accord collectif au sein des sociétés composant le groupe Le Monde relatif à la clause de cession des journalistes.

65. Les journalistes titularisés occupaient des postes en tant que précaires et ne peuvent donc être comptabilisés comme une nouvelle « force de travail ». 
La modification de la structure capitalistique du groupe modifie les modalités d'action des salariés. C'est donc à eux d'en inventer des nouvelles pour renouveler leurs capacités d'action.

$$
* * *
$$

Le processus de recapitalisation du groupe Le Monde, qui s'est achevé en novembre 2010, a mis au jour les limites du pouvoir actionnarial dans une entreprise. Ces limites tiennent à la difficulté de sortir d'un registre d'argumentation portant principalement sur le contrôle du capital, en conduisant finalement les salariés à adopter une vision patrimoniale de l'entreprise. Dans le même temps, cet attachement au contrôle du capital interdit de revenir sur l'activité de l'entreprise et les raisons pour lesquelles le journal phare du groupe ne se vend pas suffisamment pour assurer un équilibre financier. Il en résulte que, du moins dans un premier temps, les difficultés économiques sont envisagées comme des difficultés financières, dont la solution est trouvée dans la réduction du nombre de salariés, selon une arithmétique purement comptable. Sous l'égide de ce pouvoir actionnarial, les initiatives des instances représentatives du personnel se limitent alors à la recherche des incitations indemnitaires permettant d'atteindre le nombre d'emplois à supprimer sur la base d'un «volontariat» comme lors des suppressions d'emplois de 2008.

En s'engageant dans la recherche d'un « véritable patron », les sociétés de personnels, et particulièrement la SRM, ouvrent la voie à un renouvellement de l'action des comités d'entreprise dans la négociation des conditions mêmes de reprise du journal, en allant jusqu'à envisager la reconfiguration des titres du groupe, en concertation avec leurs salariés. Cette orientation nouvelle, constitutive selon nous d'un pouvoir salarial, fournit une explication aux choix de trouver un repreneur pour faire face à la crise de 2010. En abandonnant la question du contrôle du capital, et donc, corollairement, celle de l'indépendance à l'égard de l'argent, la SRM est amenée à descendre de sa position dominante au sein de l'actionnariat du groupe, pour entrer véritablement dans le contenu et la présentation du journal au côté des autres sociétés de personnels. Progressivement, le pouvoir actionnarial est délaissé par les salariés, notamment face à l'expérience d'un pouvoir patronal, pour tendre vers un pouvoir salarial, où l'enjeu se trouve dans la capacité d'intervention des salariés sur les évolutions et les transformations des produits qu'ils réalisent par leur travail.

Ce cas nous permet d'alimenter les réflexions autour d'un statut de l'entreprise de presse. Comme on l'a vu, la revendication d'un pouvoir actionnarial par les journalistes du Monde, puis par l'ensemble des per- 
sonnels du groupe, répond à la volonté d'assurer l'indépendance des salariés dans leur mission d'intérêt public qu'est l'information. Cependant, ce cas montre que le pouvoir actionnarial n'est pas la seule façon d'accéder à l'indépendance, dans la mesure où le droit du travail apporte également des éléments permettant aux salariés de s'exprimer dans l'entreprise. L'actionnariat salarié n'est ainsi pas le seul moyen pour instituer la démocratie dans l'entreprise, surtout s'il entraîne un délitement du pouvoir salarial. Il s'agit alors de refonder les pouvoirs dans l'entreprise de manière à ce que la responsabilité ne dérive pas seulement du droit de propriété (d'actionnaires) mais qu'elle soit partagée entre l'ensemble des parties prenantes ${ }^{66}$. Pour que s'institue une réelle participation des travailleurs au bon fonctionnement de leur entreprise, il faut qu'ils aient à leur disposition des droits mais aussi la capacité effective de les appliquer. Ainsi, le rachat du groupe Le Monde, s'il signe l'échec de l'actionnariat salarié, ne remet pas en cause le bien fondé de la participation des salariés dans l'entreprise, en inventant de nouvelles façons de participer.

IDHE-ENS Cachan Camille.dupuy@ens-cachan.fr

$* \quad * \quad *$

66. Comme le proposent Roger BAUDOIN et al., in L'entreprise : formes de la propriété et responsabilité sociale, op. cit. 


\section{Repères chronologiques}

\begin{tabular}{|c|c|}
\hline $\mathbf{2 0 1 0}$ & \\
\hline 28 mars & $\begin{array}{l}\text { Comité de groupe. Annonce de la recapitalisation (50 à } 100 \text { millions d'eu- } \\
\text { ros). } \\
\text { Début de la négociation sur le pôle d'indépendance. }\end{array}$ \\
\hline Avril & \\
\hline 9 Avril & $\begin{array}{l}\text { Ouverture des négociations entre les sociétés de personnels du groupe et } \\
\text { les investisseurs qui envisagent de participer à la recapitalisation. }\end{array}$ \\
\hline 18 juin & $\begin{array}{l}\text { Réunion extraordinaire du comité d'entreprise. Ouverture de la procédure } \\
\text { d'information-consultation. }\end{array}$ \\
\hline 21 juin & Deux offres fermes sont déposées : BNP et POP. \\
\hline 24 juin & $\begin{array}{l}\text { Audition des deux offres par les comités d'entreprise et les sociétés de } \\
\text { personnels. }\end{array}$ \\
\hline 25 juin & $\begin{array}{l}\text { Vote des personnels sur les offres. L'offre BNP est plébiscitée par les per- } \\
\text { sonnels. }\end{array}$ \\
\hline 28 juin & $\begin{array}{l}\text { Vote en faveur de BNP du conseil de surveillance de LMPA. Retrait de } \\
\text { l'offre POP. }\end{array}$ \\
\hline 29 juin & $\begin{array}{l}\text { Début de la négociation exclusive avec BNP (pour } 3 \text { mois) contre une } \\
\text { avance de trésorerie de } 10 \text { millions d'euros. } \\
\text { Réunion du comité d'entreprise. }\end{array}$ \\
\hline 30 juin & $\begin{array}{l}\text { Ouverture des négociations tripartites entre les repreneurs, la direction du } \\
\text { groupe et le pôle d'indépendance. }\end{array}$ \\
\hline Juillet & \\
\hline 2 juillet & $\begin{array}{l}\text { Réunion du comité d'entreprise. Présentation du projet BNP par Niel et } \\
\text { Dreyfus. }\end{array}$ \\
\hline Septembr & \\
\hline 14 sept. & Avis des comités d'entreprise de tous les titres du groupe. \\
\hline 27 sept. & $\begin{array}{l}\text { Conseil de surveillance et convocation des AG de salariés qui doivent se } \\
\text { prononcer sur la perte de contrôle. }\end{array}$ \\
\hline $\begin{array}{l}28 \text { sept. } \\
\text { Novembre }\end{array}$ & Fin de la période de négociation exclusive. \\
\hline $\begin{array}{l}2 \text { nov. } \\
\text { Décembre }\end{array}$ & Closing. Le pôle voit officiellement le jour. \\
\hline 15 déc. & $\begin{array}{l}\text { «Chasse aux coûts » avec Michaël Boukobza. } \\
\text { Ouverture de la clause de cession. } \\
\text { Conseil de surveillance du groupe : éviction de Fottorino et Guiraud. } \\
\text { Nomination de Louis Dreyfus aux deux fonctions. Démission de Louis } \\
\text { Schweitzer de la présidence du conseil de surveillance. }\end{array}$ \\
\hline 2011 & \\
\hline Février & Conseil de surveillance du groupe. \\
\hline 7 février & $\begin{array}{l}\text { J.-F. Bretelle (bras droit de Pierre Bergé) nommé président du CS. } \\
\text { Candidature de Erik Izraelewicz comme directeur du journal. }\end{array}$ \\
\hline 10 février & Proposition acceptée par la rédaction. \\
\hline & Fermeture de la clause de cession. \\
\hline
\end{tabular}

